\title{
RESEARCH
}

Open Access

\section{Balanced oral pathogenic bacteria and probiotics promoted wound healing via maintaining mesenchymal stem cell homeostasis}

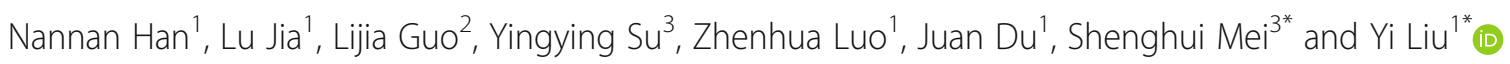

\begin{abstract}
Objectives: The homeostasis of oral pathogenic bacteria and probiotics plays a crucial role in maintaining the well-being and healthy status of human host. Our previous study confirmed that imbalanced oral microbiota could impair mesenchymal stem cell (MSC) proliferation capacity and delay wound healing. However, the effects of balanced oral pathogenic bacteria and probiotics on MSCs and wound healing are far from clear. Here, the balance of pathogenic bacteria Porphyromonas gingivalis and probiotics Lactobacillus reuteri extracts was used to investigate whether balanced oral microbiota modulate the physiological functions of MSCs and promote wound healing.
\end{abstract}

Methods: The effects of balanced pathogenic bacteria P. gingivalis and probiotics L. reuteri extracts on gingival MSCS (GMSCs) were tested using the migration, alkaline phosphatase activity, alizarin red staining, cell counting kit-8, realtime PCR, and western blot assays. To investigate the role of balanced pathogenic bacteria $P$. gingivalis and probiotics L. reuteri extracts in the wound of mice, the wounds were established in the mucosa of palate and were inoculated with bacteria every 2 days.

Results: We found that the balance between pathogenic bacteria and probiotics enhanced the migration, osteogenic differentiation, and cell proliferation of MSCs. Additionally, local inoculation of the mixture of $L$. reuteri and $P$. gingivalis promoted the process of wound healing in mice. Mechanistically, we found that LPS in $P$. gingivalis could activate NLRP3 inflammasome and inhibit function of MSCs, thereby accelerating MSC dysfunction and delaying wound healing. Furthermore, we also found that reuterin was the effective ingredient in L. reuteri which maintained the balance of pathogenic bacteria and probiotics by neutralizing LPS in P. gingivalis, thus inhibiting inflammation and promoting wound healing.

Conclusions: This study revealed that the homeostasis of oral microbiomes played an indispensable role in maintaining oral heath, provided hopeful methods for the prevention and treatment of oral diseases, and had some referential value for other systemic diseases caused by dysfunction of microbiota and MSCS.

Keywords: Oral microbiomes, MSCs, Wound healing, P. gingivalis, L. reuteri

\footnotetext{
*Correspondence: meishenghui1983@126.com; lililiuyi@163.com

${ }^{3}$ Beijing Tiantan Hospital, Capital Medical University, Beijing, People's

Republic of China

'Laboratory of Tissue Regeneration and Immunology and Department of

Periodontics, Beijing Key Laboratory of Tooth Regeneration and Function

Reconstruction, School of Stomatology, Capital Medical University, Tian Tan

Xi Li No.4, Beijing 100050, People's Republic of China

Full list of author information is available at the end of the article
}

(c) The Author(s). 2020 Open Access This article is distributed under the terms of the Creative Commons Attribution 4.0 International License (http://creativecommons.org/licenses/by/4.0/), which permits unrestricted use, distribution, and reproduction in any medium, provided you give appropriate credit to the original author(s) and the source, provide a link to the Creative Commons license, and indicate if changes were made. The Creative Commons Public Domain Dedication waiver (http://creativecommons.org/publicdomain/zero/1.0/) applies to the data made available in this article, unless otherwise stated. 


\section{Introduction}

The oral cavity, as an extension of an external body part, is actually colonized by billions of bacteria, fungi, and viruses, now known as oral microbiomes. It is fairly well known that oral microbial community includes pathogenic bacteria and probiotics, and that homeostasis of oral microbiomes plays a crucial role in maintaining the well-being and healthy status of human host [1-6]. Strong evidence has shown that once the balance of oral microbiota is broken, predominant pathogens can lead to a variety of oral diseases including periodontitis, caries, odontogenic infections, and oral mucositis [7-9]. Furthermore, several studies seem to suggest that Porphyromonas gingivalis ( $P$. gingivalis) may be a driving factor in the development of oral and digestive cancer, such as oral squamous cell carcinoma and intestinal and pancreatic cancer $[10,11]$. It is now widely accepted that alterations in the dysbiosis of the oral microbiota may influence the normal host-microbe crosstalk, thus resulting in a higher risk for the onset of diseases. Probiotics are a kind of microorganisms which are beneficial to the health of the host and can produce health care effects when given to the host in proper dose. Probiotics, including Lactobacillus, Bifidobacteria, Escherichia coli, and Enterococcus faecalis, mainly keep the health of body by maintaining the balance of host bacteria, secreting antimicrobial substances, and modulating immune response [12]. Previous studies have shown that probiotics can inhibit the proliferation and adhesion of oral cariogenic bacteria by colonizing in oral cavity and producing metabolites. In addition, related studies have found that oral probiotics can also improve the halitosis by adjusting oral flora and reducing volatile sulfides. Oral candidiasis is a common oral mucosal infectious disease. In vitro experiments have demonstrated that probiotics can inhibit the proliferation, growth, and adhesion of Candida albicans and reduce the formation of biofilm $[3,13]$. Therefore, the balance between pathogenic bacteria and probiotics is very indispensable to maintain oral health.

Oral wounds are commonly caused by surgical excision of lesions, vulnus, recurrent ulcer, and radiation injury, and usually accompanied by oral mucosal and soft tissue defects, which can lead to scar formation and tissue adhesion. It has been reported that wound healing process is delayed as a result of disturbances of microbiota adhesion at the sites of wounds [14-17]. Mesenchymal stem cells (MSCs) are considered as a promising method that has the potential to promote tissue regeneration and wound healing due to the multilineage differentiation and self-renewal properties [18-21]. Our previous study found that in an environment of oral microecological imbalance, the function of oral gingival and palatal MSCs was impaired, which played an important role in the repair of oral soft tissue damage, and the healing speed of oral soft tissue was significantly slowed down [22]. However, the effects of the balance between oral pathogenic bacteria and probiotics on the physiological function of MSCs and wound healing are still unclear.

In this work, we investigated the role of the balance of oral pathogenic bacteria and probiotics in the regulation of MSCs' potentials and wound healing. Our results revealed that simulating the balance between oral pathogenic bacteria and probiotics with sonicated extracts from $L$. reuteri and $P$. gingivalis could activate the migration, osteogenic differentiation, and proliferation of GMSCs in vitro. Furthermore, we also found that reuterin was the effective ingredient in L. reuteri which maintained the balance of oral pathogenic bacteria and probiotics by neutralizing LPS in $P$. gingivalis. Additionally, the local inoculation of the mixture of $L$. reuteri and $P$. gingivalis restored the process of wound healing in mice. Our findings are based on the concept of the balance of oral microecology, which provides promising ideas and methods for the prevention and treatment of oral diseases, and can be used for reference in other systemic diseases caused by dysfunction of microbiota and MSCs.

\section{Materials and methods Animals}

Eight-week-old female C57BL/6 mice were gained from SPF Biotechnology Company (Beijing, China). Mice were raised under the standard conditions following the Animal Care and Use Committee of Capital Medical University. All animal researches were abided by the rules approved by the Beijing Stomatological Hospital, Capital Medical University (Ethical Committee Agreement, Beijing Stomatological Hospital Ethics Review No. KQYY-201710-001).

\section{Cell cultures}

Gingival tissues were isolated from C57BL/6 mice. Solutions of $75 \%$ ethanol and phosphate-buffered saline (PBS) were used to disinfect and rinse the tissues. After that, gingival tissues were digested by a solution of $3 \mathrm{mg} / \mathrm{ml}$ collagenase type I (Sigma-Aldrich, USA) and $4 \mathrm{mg} / \mathrm{ml}$ dispase (Sigma-Aldrich, USA) for $1 \mathrm{~h}$ at $37^{\circ} \mathrm{C}$. A $70-\mu \mathrm{m}$ strainer (Falcon, USA) was used to filter dissociated GMSC suspensions. GMSCs were cultivated in a humidified incubator under $5 \% \mathrm{CO}_{2}$ at $37^{\circ} \mathrm{C}$ in DMEM alpha modified Eagle's medium (Invitrogen, USA), renewal with $20 \%$ fetal bovine serum (FBS; Invitrogen), $100 \mu \mathrm{g} / \mathrm{ml}$ streptomycin, $100 \mathrm{U} / \mathrm{ml}$ penicillin, and $2 \mathrm{mmol} / \mathrm{l}$ glutamine (Invitrogen).

\section{Bacterial strain culture and preparation of bacterial extracts}

Porphyromonas gingivalis (P. gingivalis) ATCC 33277 and Lactobacillus reuteri (L. reuteri) ATCC 11284 were grown on agar plates at $37^{\circ} \mathrm{C}$ in an anaerobic chamber 
with MRS (AOBOX, Beijing) for 5-7 days. For experiments in vitro on GMSCs, Lactobacillus reuteri cells were scraped and resuspended in $10 \mathrm{ml}$ phosphatebuffered solution (PBS), sonicated (30 min, $300 \mathrm{~W}, 10 \mathrm{~s}$ sonication, and $10 \mathrm{~s}$ pause) with ultrasonic crushing apparatus (JYD-150, China) on an ice bath. The sonicated preparation was centrifuged at $14,000 \mathrm{rpm}$ for $20 \mathrm{~min}$ at $4{ }^{\circ} \mathrm{C}$ and discarded the supernatants; the weight of precipitates was taken and diluted with sterile deionized water (bacterial extracts) [23]. Bacterial extracts reached a certain concentration and filtered with $0.22-\mu \mathrm{m}$ strainer (Falcon, USA), stored at $20{ }^{\circ} \mathrm{C}$ until used. For animal experiments in vivo, bacteria ( $P$. gingivalis and $L$. reuteri) should be cultured way in advance. On the day of treatment, bacteria were harvested by centrifugation, washed three times with PBS, and quantified using spectrophotometer. (the number of $P$. gingivalis at OD of 0.8 was approximately $1 \times 10^{9} / \mathrm{ml}$ ). Bacteria were resuspended in $2 \%$ methylcellulose (Sigma Aldrich, USA) $\left(2 \times 10^{9} / 100 \mu \mathrm{l} /\right.$ mouse $)$.

\section{Cell scratch migration assays}

GMSCs were cultured on six-well plates as confluent monolayers, a cross wound was scratched using a 200- $\mu \mathrm{l}$ pipet tip (Axygen ${ }^{\circ}$ Corning, NY, USA), and then, cells were cultured in fresh culture media without fetal bovine serum. To observe the extent of wound migration, images were taken using microscopy at baseline $(0 \mathrm{~h}), 24 \mathrm{~h}$, $48 \mathrm{~h}$, and $72 \mathrm{~h}$ after scratching. The void area (VA) of wound was measured by Image-Pro (National Institutes of Health, USA), and the height and the relative width were calculated $($ Area $\%=V A /$ height $)$.

Real-time reverse transcriptase-polymerase chain reaction Total RNA was isolated from GMSCs using TRIzol reagents (Invitrogen, USA) and reverse transcribed into cDNA following the manufacturer's protocol (Takara, Dalian, China). Then, real-time RT-PCR reactions were performed using the SYBR Premix Ex TaqTM (Takara, Dalian, China) and an Icycler iQ Multi-colour Real-time RT-PCR Detection System. The primers for specific genes are listed in Additional file 1: Table S1.

\section{Alkaline phosphatase and alizarin red detection}

To detect the abilities of GMSC osteogenesis and mineralization, we used alizarin red staining and alkaline phosphatase activity assays according to the StemPro osteogenesis differentiation kit (Invitrogen, USA) and the manufacturer's protocol of ALP activity (Sigma-Aldrich, USA).

\section{Oil Red $\mathrm{O}$ staining analysis}

GMSCs were cultivated on six-well plates and treated with $P$. gingivalis and $L$. reuteri sonic extracts respectively in lipogenic induction medium (Invitrogen, USA) for 1 month. Then, the medium was removed, added with $2 \mathrm{ml}$ of $10 \%$ formalin, and incubated for $10 \mathrm{~min}$ at room temperature. Cells were washed with $2 \mathrm{ml}$ of $60 \%$ isopropanol for $5 \mathrm{~min}$, and let the cells dry completely. One milliliter of Oil Red $\mathrm{O}$ working solution was added and incubated at RT for $10 \mathrm{~min}$. Images were acquired under the microscope for analysis. Oil Red O dye (Sigma, USA) was eluted by adding $1 \mathrm{ml}$ of 100\% isopropanol and incubated for $10 \mathrm{~min}$ with gentle shaking. OD value was measured at $500 \mathrm{~nm}$ using $100 \%$ isopropanol as blank.

\section{Western blot analysis}

GMSCs were lysed using RIPA buffer. The details of method for western blot were depicted as previously [24]. The expressions of protein were detected using anti-NLRP3 (1:300, Abcam), and $\beta$-actin (1:2000, Abcam) was used as an internal control. The secondary antibodies were obtained from the commercial companies: anti-mouse IgG (1:2000, Abcam) and anti-rabbit IgG (1:5000, Abcam).

\section{$16 \mathrm{~S}$ rRNA sequencing and analysis}

An antibiotic cocktail of $1300 \mathrm{mg} / \mathrm{l}$ of metronidazole and $660 \mathrm{mg} / \mathrm{l}$ of levofloxacin in the drinking water was used to remove oral bacteria in mice for 2 weeks. After that, the bacteria of oral mucosa of mice were scraped with cotton swab and analyzed as previously [22]. UCLUST was used to divide the unique sequence set into operational classifiers (OTUs) under 97\% recognition threshold. Student's $t$ test was implemented to evaluate alpha and beta diversity. The Kruskal-Wallis test was used to test the significance of classified variables.

\section{Wound healing mouse model}

C57BL/6 mice were anesthetized by intraperitoneal injection of $1 \%$ chloral hydrate using $1 \mathrm{ml}$ syringe. Chloral hydrate was injected based on the weight of the mice; the delivered dose is chloral hydrate $400 \mathrm{mg} / \mathrm{kg}$. The wounds were established in the mucosa of palate, and the region of wound was from the mesial margin of the first molar to the distal of the third molar; full thickness mucosa was removed. Mice with wound were randomly distributed to four groups: incubation of $P$. gingivalis group, incubation of $L$. reuteri group, incubation of $P$. gingivalis and $L$. reuteri group, or untreated group. The wounds were inoculated with bacteria every 2 days. To observe the extent of wound healing, mice were sacrificed after incubation for 7 and 14 days. Stereomicroscopy and Image $J$ were used to measure the area of wound healing. Then, the maxillary palates were fixed in $4 \%$ paraformaldehyde for $48 \mathrm{~h}$, and all samples were decalcified with buffered 10\% EDTA and embedded in 
paraffin. Then, all samples were deparaffinized and stained with hematoxylin and eosin.

\section{Statistics}

The SPSS10 software was used to analyze all statistical computations. Statistical significance was identified by Student's $t$ test, Duncan's test, or one-way ANOVA, with a $P \leq 0.05$ regarded as significant.

\section{Results}

The balance between pathogenic bacteria and probiotics restored GMSC migration

To investigate the balance of oral pathogenic bacteria and probiotics in vitro, we performed scratch-simulated wound migration assay in GMSCs. In the first place, different concentrations of the $P$. gingivalis sonicated extracts were added to the cells; according to cell scratch migration assay results, we found that $50 \mu \mathrm{g} / \mathrm{ml} \mathrm{P.} \mathrm{gingi-}$ valis sonicated extracts significantly inhibited GMSC migration at $24 \mathrm{~h}$ and $48 \mathrm{~h}$ (Fig. 1a, b). Secondly, to investigate the effect of probiotics on the migration of GMSCs, we discovered that $50 \mu \mathrm{g} / \mathrm{ml}$ L. reuteri sonicated extracts markedly promoted GMSC migration (Fig. 1c, d). Finally, according to the above screened concentration of $P$. gingivalis and $L$. reuteri sonicated extracts, respectively, on the basis of $50 \mu \mathrm{g} / \mathrm{ml} P$. gingivalis sonicated extracts, we mixed different proportions of pathogenic bacteria and probiotics into the GMSCs; the cell scratch migration results demonstrated that the ratio of $P$. gingivalis bacteria extracts to $L$. reuteri sonicated extracts was 1 to 0.5 (1, $50 \mu \mathrm{g} / \mathrm{ml} \mathrm{P}$. gingivalis sonicated extracts; $0.5,25 \mu \mathrm{g} / \mathrm{ml} L$. reuteri sonicated extracts), which restored GMSC migration (Fig. 1e, f). Next, the ratio of 1 to 0.5 was used to mimic the balance of oral pathogenic bacteria and probiotics in all experiments in vitro.

\section{The balance between pathogenic bacteria and probiotics} restored the function of GMSCs

To test the effect of the balance between pathogenic bacteria and probiotics on GMSCs, the cells were treated with $50 \mu \mathrm{g} / \mathrm{ml} P$. gingivalis sonicated extracts and the mixture of $P$. gingivalis bacteria extracts and $L$. reuteri sonicated extracts. The osteogenic differentiation abilities of GMSCs were evaluated using ALP activity assay, the cells were transduced in osteogenic-inducing medium for 5 days, and we found that the ratio of $P$. gingivalis sonicated extracts to $L$. reuteri sonicated extracts was 1 to 0.5 , which rescued the ALP activity of GMSCs (Fig. 2a). Three weeks after osteogenic induction, the alizarin red staining assay results showed that the balance between pathogenic bacteria and probiotics rescued the osteogenic differentiation capacity which was impaired by $P$. gingivalis sonicated extract treatment (Fig. 2b). In addition, to further test the impact of microbiome balance on osteogenic GMSCs, we performed real-time RT-PCR to verify the expression of the crucial transcription factors for regulating osteogenic differentiation: OSX, OPN, OCN, and RUNX2, and our results demonstrated that the ratio of $P$. gingivalis

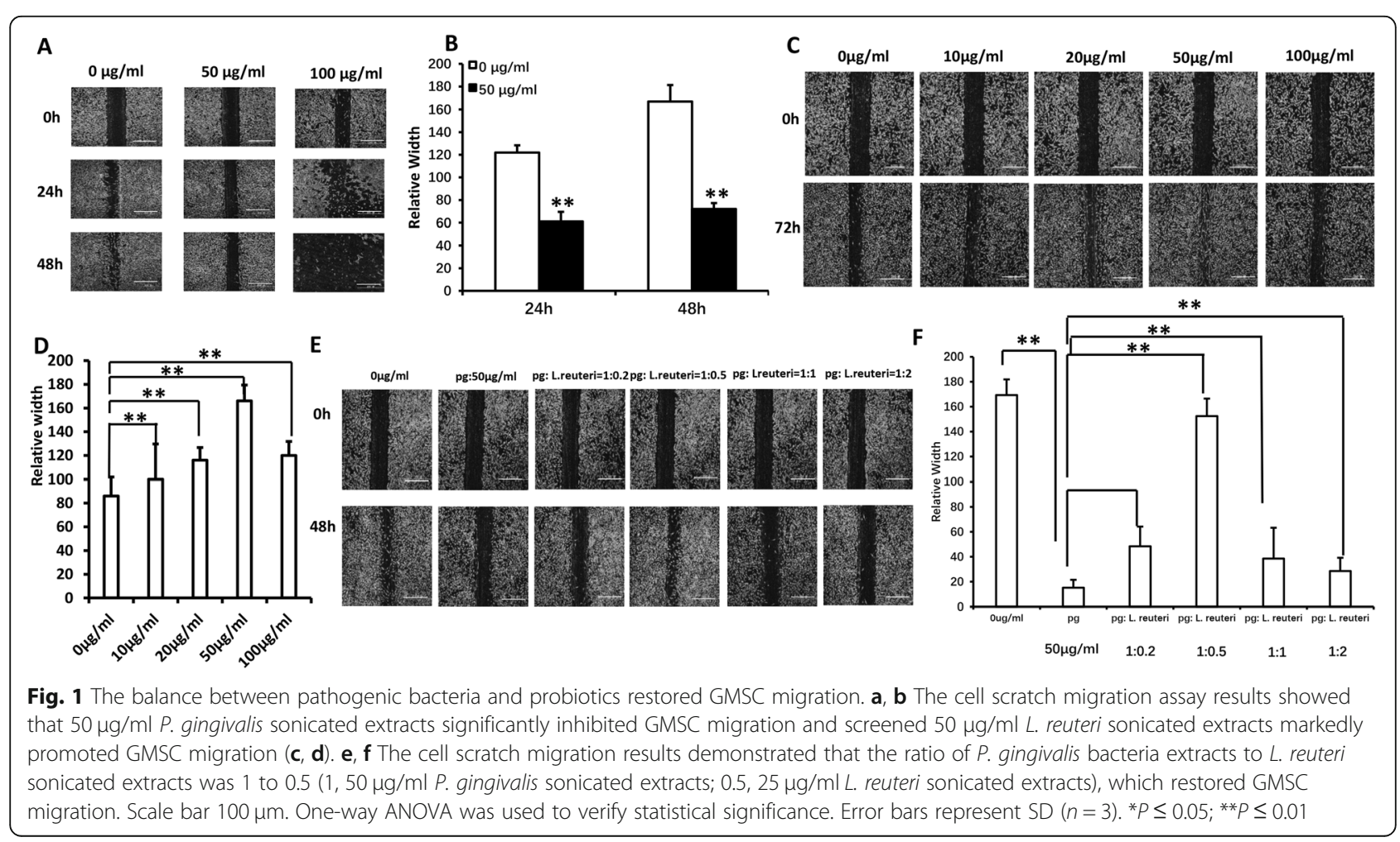




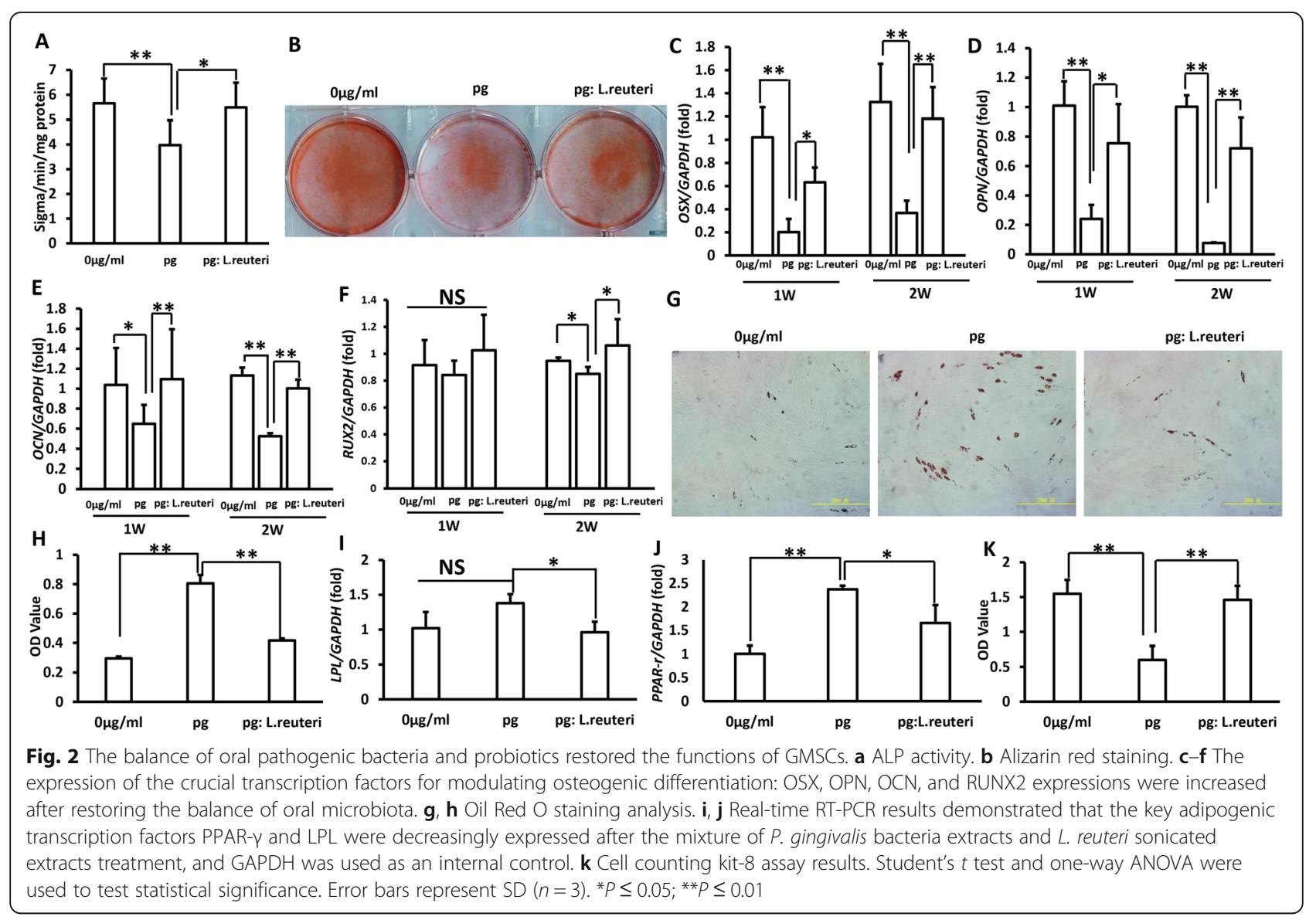

sonicated extracts to $L$. reuteri sonicated extracts was 1 to 0.5 , which restored the expressions of OSX, OPN, OCN, and RUNX2 (Fig. 2c-f). Oil Red $\mathrm{O}$ staining analysis results showed that the balance between pathogenic bacteria and probiotics inhibited the GMSC adipogenic differentiation caused by $P$. gingivalis sonicated extracts (Fig. $2 \mathrm{~g}, \mathrm{~h}$ ). Meanwhile, real-time RT-PCR results demonstrated that the key adipogenic transcription factors $P P A R-\gamma$ and $L P L$ were decreasingly expressed after the mixture of $P$. gingivalis bacteria extract and $L$. reuteri sonicated extract treatment (Fig. 2i, j). The cell counting kit-8 assay results showed that he ratio of $P$. gingivalis sonicated extracts to L. reuteri sonicated extracts was 1 to 0.5 , which rescued the proliferation capacity of GMSCs compared to the $P$. gingivalis sonicated extract group (Fig. 2k). These findings elucidated that the balance between pathogenic bacteria and probiotics could maintain the functions of GMSCs.

\section{The oral predominant pathogenic bacteria in mice decreased after antibiotic treatment}

To analyze the variation of oral bacterial communities in mice after antibiotic treatment, we collected oral microbiota from oral mucosa tissue samples, and $16 \mathrm{~S}$ rRNA gene sequencing results demonstrated that the differences of a general discrete clustering pattern of bacterial taxa were observed between mice with antibiotic treatment and control group (Fig. 3a). Barplot analysis further revealed that the bacterial relative abundance was significantly decreased in antibiotic treatment group compared with control group (Fig. 3b). LefSe analysis results showed that the predominant pathogenic bacteria decreased after antibiotic treatment (Fig. 3c). On the basis of UPGMA analysis, we further demonstrated that the microbial communities were reduced in antibiotics group (Fig. 3d), and these findings suggested that the predominant pathogenic bacteria decreased after antibiotic treatment.

\section{The balance of pathogenic bacteria and probiotics promoted wound healing process in mice}

To further verify the effect of the balance of oral pathogenic bacteria and probiotics on wound, we established wound healing model of palate in mice after antibiotic treatment (Fig. 4a, e). The wounds were inoculated with either $P$. gingivalis $\left(2 \times 10^{9} \mathrm{CFU}\right.$ in $2 \%$ methylcellulose $200 \mu \mathrm{l})$ or the mixture of $L$. reuteri and P. gingivalis, or untreated group. Each group was inoculated once every 2 days. The mice were sacrificed after inoculation for 7 and 14 days, and the area of wound healing was observed 


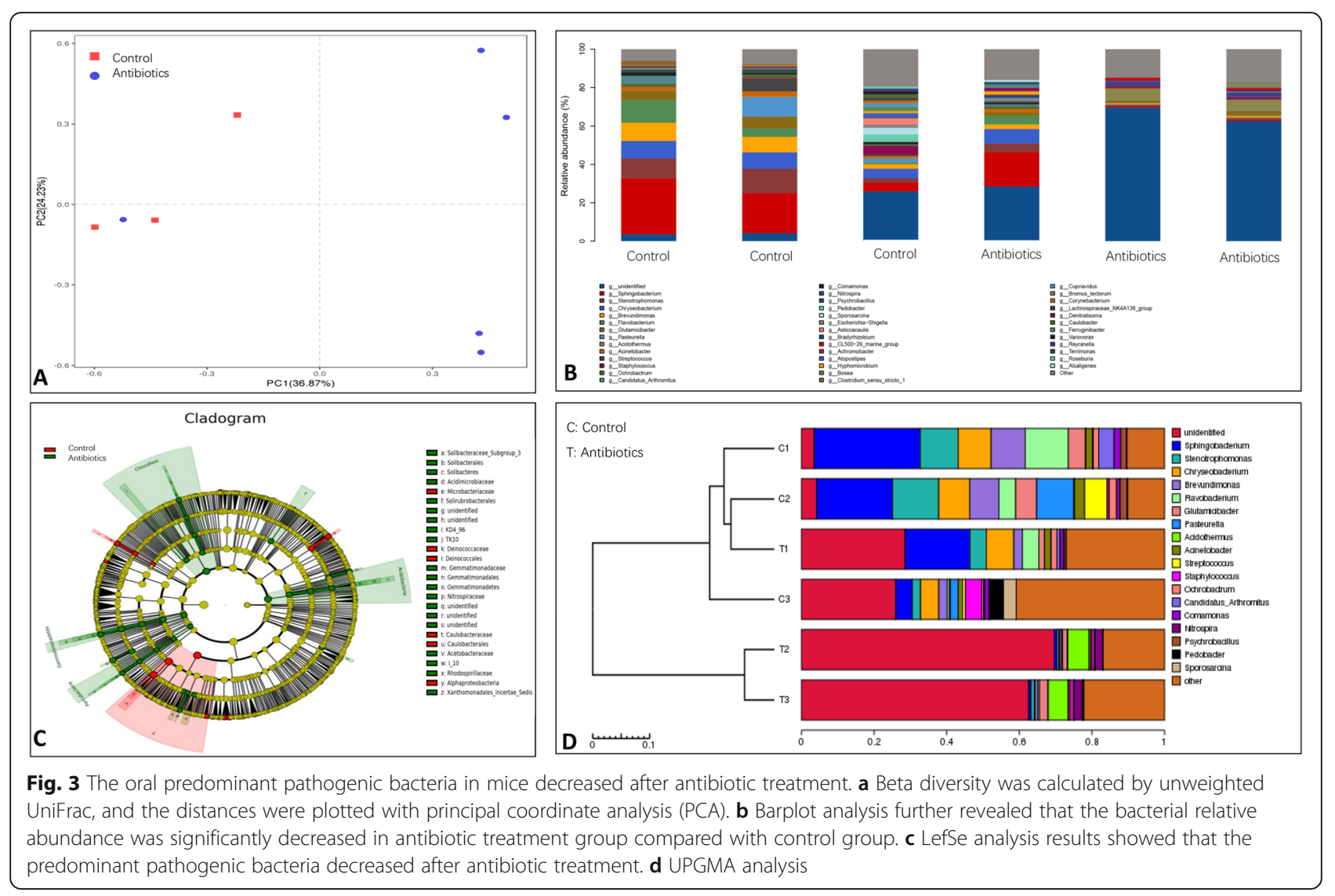

by stereomicroscopy and measured using Image J. We discovered that inoculation of the mixture of $L$. reuteri and P. gingivalis group promoted wound healing (Fig. 4c, g), while the wound healing process was delayed in the inoculation of $P$. gingivalis group (Fig. $4 \mathrm{~b}, \mathrm{f}$ ), and the wound healing in the untreated group was slower than the mixture of L. reuteri and P. gingivalis group (Fig. $4 \mathrm{~d}$, h). In addition, we assessed the quantity of the unhealed area of wound; the results showed statistically significant differences in the $P$. gingivalis inoculation group, in the mixture of $L$. reuteri and $P$. gingivalis group, and in the untreated group (Fig. 4i, j), suggesting that the balance of oral microbiome promoted wound healing in mice.

Histopathological photomicrograph results further revealed that the inoculation of the mixture of $L$. reuteri and P. gingivalis group (Fig. $5 \mathrm{c}, \mathrm{g}$ ) markedly accelerated wound healing compared to the $P$. gingivalis group (Fig. 5b, f) and untreated group (Fig. 5d, h). Furthermore, the length of the wound was measured using Image J, we found that the length of wound was shorter in the mixture of $L$. reuteri and $P$. gingivalis group than in the inoculation of $P$. gingivalis group and untreated group (Fig. 5i, j), and there were significant statistical differences in these three groups. These findings further demonstrated that the balance of oral microbiome played a crucial role in wound healing process.
LPS increased in $P$. gingivalis and thereby inhibited the functions of MSCs by activating NLRP3 inflammasome It has been reported that NLRP3 inflammasome played an important role in immune response and a wide variety of disease occurrence [25-27]. To identify if the underlying molecular mechanisms of $P$. gingivalis inhibited the functions of MSCs, we tested the expression of NLRP3 from the wound tissues of palate in mice after inoculation with bacteria; western blot and real-time RT-PCR results revealed that NLRP3 expression was significantly increased in the inoculation of $P$. gingivalis group compared to the mixture of $L$. reuteri and $P$. gingivalis group, and untreated group (Fig. 6a, b). Next, we used exogenous LPS as NLRP3 activator and CY-09 as NLRP3 inhibitor to test the functions of GMSCs [28]; the cell migration assay results showed that LPS significantly inhibited GMSC migration; however, NLRP3 inhibitor CY-09 rescued the capacity of GMSC migration after LPS treatment (Fig. 6c, d). This suggested that LPS could activate NLRP3 inflammasome. To further testify the impact of NLRP3 activation on mesenchymal stem cell markers, real-time RT-PCR results showed that LPS inhibited the expression of SOX2, OCT4, and NANOG, while CY-09 restored the capacities of MSCs (Fig. 6e-g). ALP activity assay and alizarin red staining assay results confirmed that NLRP3 activation inhibited ALP activity 

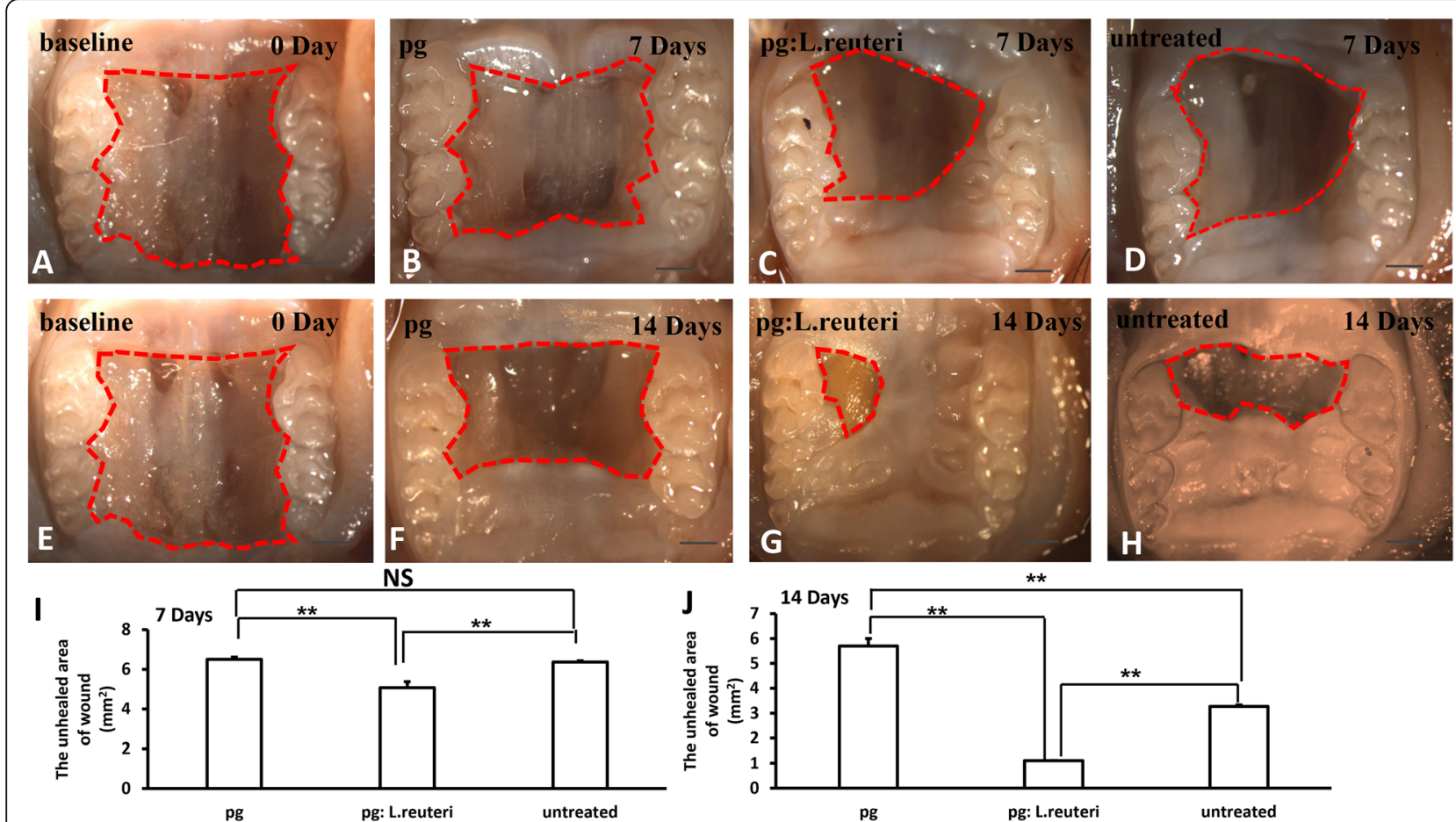

Fig. 4 The balance of oral pathogenic bacteria and probiotics promoted wound healing process in mice. a, e Macroscopic observation showed that wound healing model of palate was established in mice after antibiotic treatment. $\mathbf{b}$, $\mathbf{f}$ The inoculation of $P$. gingivalis delayed wound healing after 7 and 14 days. $\mathbf{c}, \mathbf{g}$ The inoculation of the mixture of L. reuteri and $P$. gingivalis group promoted wound healing compared with untreated group (d, h). i, j Quantitative analysis of the unhealed area of wound after inoculation for 7 and 14 days, respectively. Scale bar 1 mm. Error bars represent SD $(n=6)$. Red dotted line, the unhealed area of wound. ${ }^{*} P \leq 0.05 ;{ }^{*} p \leq 0.01$

and mineralization in GMSCs (Fig. 6h, i); moreover, real-time RT-PCR results further testified that NLRP3 activation inhibited the expression of the crucial transcription factors for modulating osteogenic differentiation: RUX2, OSX, OPN, and OCN (Fig. 6j-m), and CY-09 rescued the functions of osteogenic differentiation. In addition, counting kit- 8 assay results showed that CY-09 restored the proliferation potential of GMSCs caused by LPS-induced NLRP3 activation (Fig. 6n). On the basis of these results, we demonstrated that $P$. gingivalis inhibited the functions of MSCs by activating NLRP3 inflammasome.

\section{Reuterin maintained the balance of oral pathogenic bacteria and probiotics by neutralizing LPS}

It is well known that LPS is the main pathogenic component of gram-negative bacteria, which caused a series of oral diseases such as periodontitis, gingivitis, and inflammatory disease and impaired the functions of MSCs [29-32]. Next, to detect whether bacterial sonicated extracts existed LPS and the interaction between pathogenic bacteria and probiotics, ELISA assay was used to measure the content of LPS; our results demonstrated that a large amount of LPS existed in $P$. gingivalis sonicated extracts; however, the content of LPS was significantly decreased in the mixture of $P$. gingivalis bacteria extract and $L$. reuteri sonicated extract group (Fig. 7a). This suggested that some ingredients could neutralize LPS in L. reuteri sonicated extracts. Next, we found that LPS was obviously reduced after $P$. gingivalis bacteria extracts interacted with reuterin (Fig. 7b). Chromatography assay results further confirmed that $L$. reuteri sonicated extracts contained reuterin (Fig. 7c); thus, LPS in P. gingivalis could activate NLRP3 inflammasome resulting in inflammation and inhibit function of MSCs, thereby accelerating MSC dysfunction and delaying wound healing. When pathogenic $P$. gingivalis with L.reutri were mixed, reuterin was the effective ingredient in $L$. reuteri which maintained the balance of pathogenic bacteria and probiotics by neutralizing LPS in $P$. gingivalis, thus promoting wound healing (Fig. 7d).

\section{Discussion}

To date, an increasing number of studies have suggested that the balance of oral microbial ecosystem plays an indispensable role in maintaining human health. A shift of oral microbiota may cause both oral and systemic diseases $[33,34]$. Our previous study revealed that the imbalance of oral microbiome impaired GMSC proliferation and palatal wound healing. However, the impact of balance of oral microbial ecosystem on MSC 


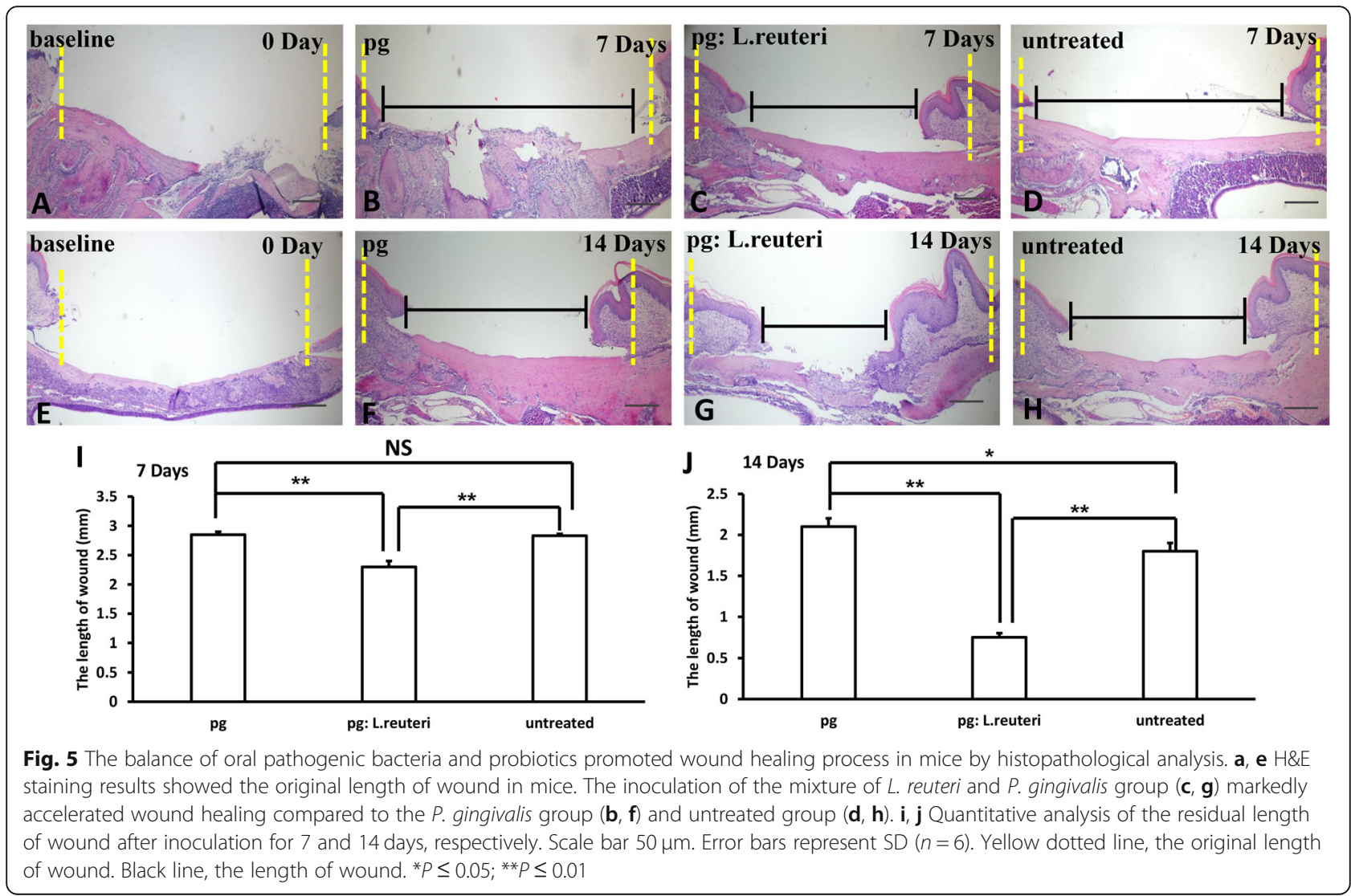

function and wound healing, and the mechanisms of interactions between oral bacterial communities remain elusive. In oral cavity, some specific bacteria, such as $P$. gingivalis, can change the proportion of other microorganisms in the ecological niche by changing the environment and are defined as key pathogen $[35,36]$. Thus, in this study, we used $P$. gingivalis as the pathogenic state, and the ratio of $P$. gingivalis to $L$. reuteri sonicated extracts was 1 to 0.5 which was used to mimic the balance of oral microbiota; we discovered that $50 \mu \mathrm{g} / \mathrm{ml} P$. gingivalis sonicated extracts significantly inhibited GMSC migration, osteogenic differentiation, and proliferation capacities, and promoted adipogenic differentiation in vitro. However, when the mixture of $L$. reuteri sonicated extracts and $P$. gingivalis bacterial extracts was used to treat GMSCs, we found that the migration, osteogenic and adipogenic differentiation, and cell proliferation potentials of GMSCs could be restored in oral microecological balance. LPS, a specific component of the cell wall of gram-negative bacteria, can induce cascade reaction of immune stimulation and toxic pathophysiological activities in the body [37-39]. Our previous study confirmed that LPS increased in mice with antibiotic treatment, which resulted in delayed wound healing and inhibited the proliferation capacity of GMSCs. In the present study, we found that $P$. gingivalis sonicated extracts contained large amounts of LPS, suggesting that LPS was a key pathogenic factor which could impair MSC function and wound healing. Moreover, we discovered that the content of LPS was significantly reduced after mixing $P$. gingivalis and $L$. reuteri sonicated extracts. $L$. reuteri not only has the main beneficial effect of Lactobacillus, it also has the ability to produce a broad spectrum of antimicrobial agent [40-42]. This substance, termed reuterin, has been reported to effectively inhibit the growth of harmful bacteria. Here, high levels of reuterin were tested in $L$. reuteri sonicated extracts, which could neutralize LPS and restore the balance between pathogenic bacteria and probiotics, and reuterin could promote the migration of GMSCs. These findings collectively suggest that reuterin maintained the balance of oral microecology by neutralizing LPS, thus restoring the function of GMSCs.

To further test the impact of the balance of oral microbiota on wound healing, we created the wound healing model in mice after antibiotic treatment. According to macroscopic observation and histopathological photomicrographs, we found that local inoculation of $P$. gingivalis 


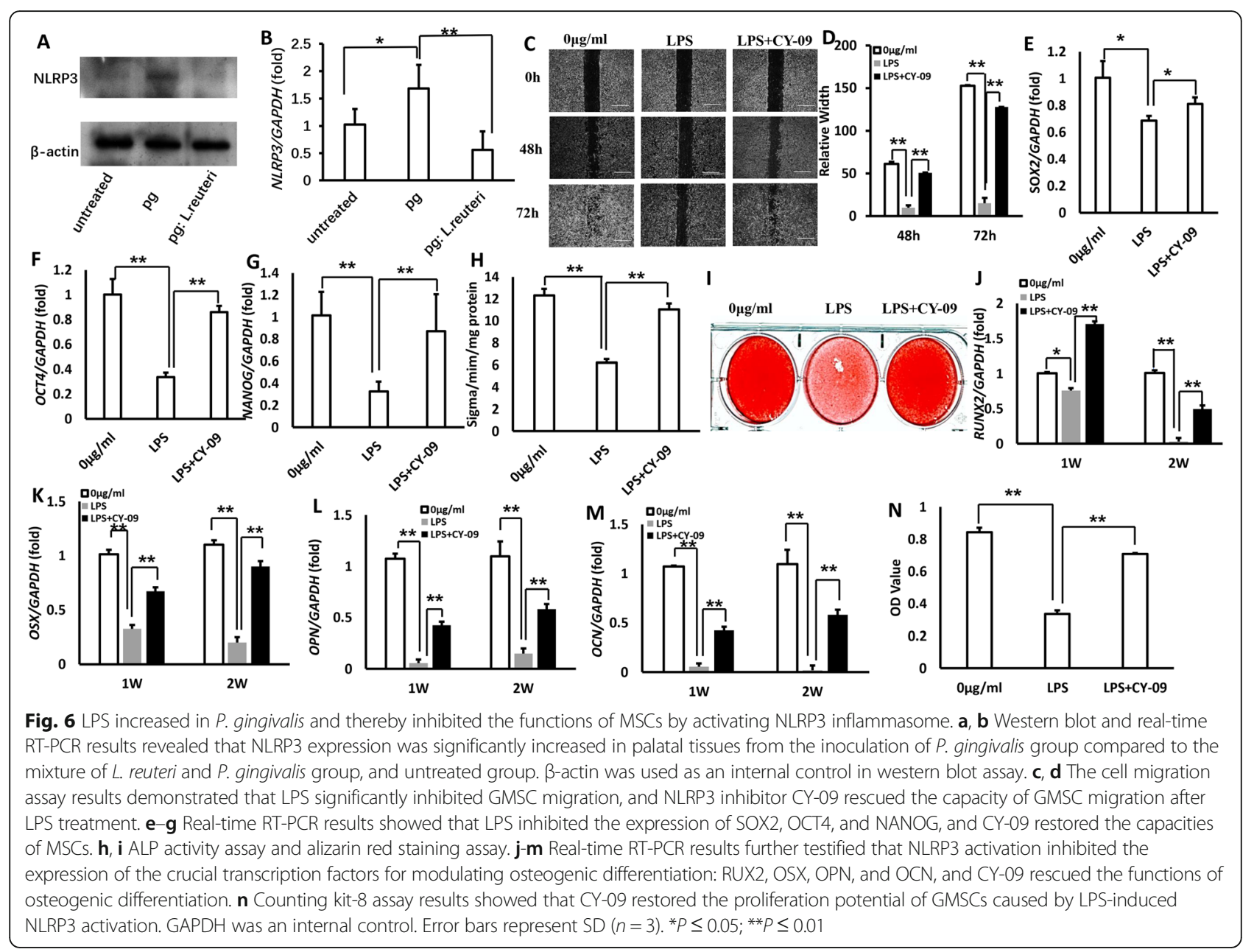

significantly inhibited wound healing; however, inoculation of the mixture of $P$. gingivalis and $L$. reuteri markedly promoted the wound healing process. In addition, histopathological photomicrographs of the wound healing model further indicated that local inoculation of the mixture of $P$. gingivalis and $L$. reuteri accelerated the wound healing in comparison with the $P$. gingivalis-inoculated and untreated groups. These data suggested that the balance of oral microbiota played an important role in promoting wound healing.

Inflammasomes are multiprotein complexes that can recognize a variety of pathogenic microorganisms and stress-related endogenous signaling molecules and play a critical role in innate immune system. As the core of inflammatory response, NLRP3 inflammasome plays a key role in many diseases, including familial periodic inflammation, type 2 diabetes mellitus, Alzheimer's disease, and atherosclerosis. It has been reported that $P$. gingivalis-LPS could activate NLRP3 inflammasome in mouse periodontal ligament fibroblasts and cause chronic periodontitis; however, the relevance of NLRP3 inflammasome activation and MSC function is far from clear [43, 44]. In this study, we provide evidence that LPS in $P$. gingivalis could activate NLRP3 inflammasome and inhibit the migration, self-renewal capacity, osteogenic differentiation, and cell proliferation of GMSCs, indicating that NLRP3 inflammasome activation could accelerate MSC dysfunction and that reuterin is critical for MSC function due to its effect on inhibition of NLRP3 inflammasome activation.

In summary, our data showed that the balance of oral microbiota can activate the functions of MSCs and that reuterin in $L$. reuteri maintained the balance of oral microecology by neutralizing LPS, thus maintaining the potentials of MSCs and promoting wound healing. Our study shows a link between the balance of oral microecology and wound healing, which suggests the importance of oral microbiome balance in sustaining MSC function and oral homeostasis. 


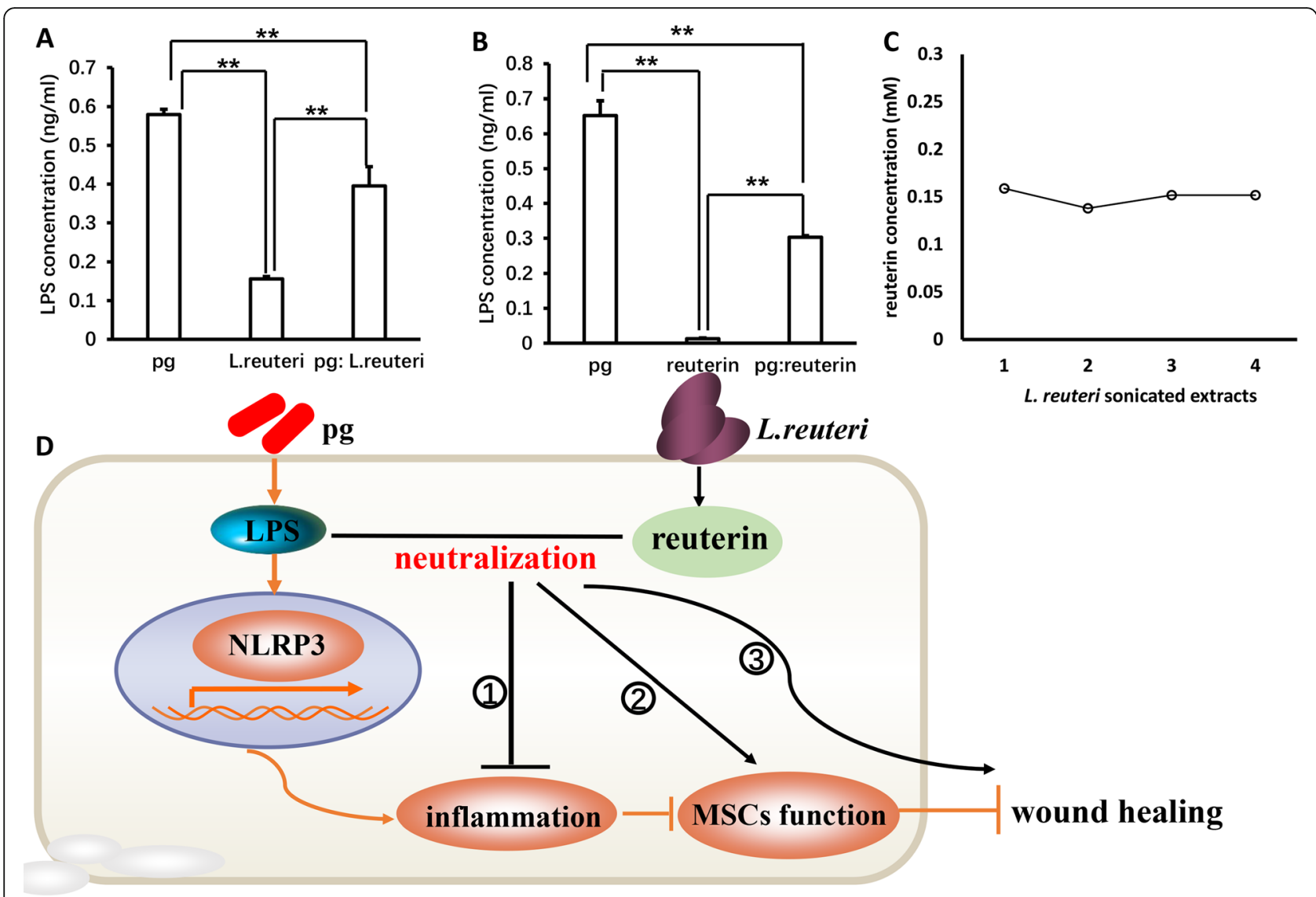

Fig. 7 Reuterin maintained the balance of oral pathogenic bacteria and probiotics by neutralizing LPS. a ELISA assay results demonstrated that a large amount of LPS existed in P. gingivalis sonicated extracts, and the content of LPS was significantly decreased in the mixture of $P$. gingivalis bacteria extract and $L$. reuteri sonicated extract group. $\mathbf{b}$ ELISA assay results showed that reuterin could neutralize LPS. c Chromatography assay results further confirmed that $L$. reuteri sonicated extracts contained reuterin, and reuterin maintained the balance of pathogenic bacteria and probiotics by neutralizing LPS in P. gingivalis, thus promoting wound healing (d). One-way ANOVA and Student's $t$ test were used to calculate statistical significance. Error bars represent SD $(n=3)$. ${ }^{*} P \leq 0.05 ;{ }^{* *} P \leq 0.01$

However, oral microbes are composed of a variety of bacteria, and we only used the mixture of $P$. gingivalis and $L$. reuteri to mimic the balance of oral microecology in this work. Future investigations will need a wider variety of bacteria to elucidate the effect of oral microbiome balance on oral health.

\section{Conclusion}

In a word, our findings revealed that simulating the balance between oral pathogenic bacteria and probiotics with sonicated extracts from $L$. reuteri and $P$. gingivalis could activate the functions of MSCs and that reuterin in L. reuteri maintained the balance of oral microecology by neutralizing LPS in P. gingivalis, thus maintaining the homeostasis of MSCs and promoting wound healing. Our findings are based on the concept of the balance of oral microecology, which provides promising ideas and methods for the prevention and treatment of oral diseases, and can be used for reference in other systemic diseases caused by dysfunction of microbiota and MSCs.

\section{Supplementary information}

Supplementary information accompanies this paper at https://doi.org/10. 1186/s13287-020-1569-2.

Additional file 1: Table S1. Primers sequences used in the real-time RT-PCR

Additional file 2: Figure S1. The balance of oral pathogenic bacteria - $P$. gingivalis and probiotics- $L$. reuteri extracts promoted wound healing in mice. (A) Macroscopic observation showed that wound healing model of palate was established in mice after antibiotics treatment. (B) The local injection of P. gingivalis extracts delayed wound healing after 7 days. (C) The local injection of the mixture of $L$. reuteri extracts and $P$. gingivalis extracts group promoted wound healing compared with untreated group (D). (E) Quantitative analysis of the unhealed area of wound after inoculation for 7 days respectively. Scale bar: $1 \mathrm{~mm}$. Error bars represent SD $(n=6)$. Red dotted line: original the area of wound, Yellow dotted line: the unhealed area of wound. ${ }^{*} P \leq 0.05 ;{ }^{* *} P \leq 0.01$.

Additional file 3: Figure S2. The expression of surface markers in GMSCs from C57BL/6 mice. (A, B, C) GMSCs were isolated from mice expressed CD146. Scale bar: $50 \mu \mathrm{m}$. (D, E) Flow cytometric analysis results (CD146 positive rate: $97.1 \%)$. (F, G, H) GMSCs were from C57BL/6 mice expressed CD90. Scale bar: $50 \mu \mathrm{m}$. (I, J) Flow cytometric analysis results (CD90 positive rate: $90.7 \%$ ). (K, L, M) CD45 were not expressed in GMSCS obtained from mice. Scale bar: $50 \mu \mathrm{m}$. (N, O) Flow cytometric analysis results (positive rate: 1.69\%). (P, Q, R) GMSCs were from C57BL/6 mice 
expressed CD44. Scale bar: 50 um. (S, T) Flow cytometric analysis results (CD44 positive rate: 87.8\%). Student's t-test was utilized for analysis in $E_{1} J$, $\mathrm{O}, \mathrm{T}$. Error bars represent SD $(n=3) .{ }^{*} P \leq 0.05 ;{ }^{*} P \leq 0.01$.

\section{Abbreviations}

ALP: Alkaline phosphatase; ARS: Alizarin red staining;

GAPDH: Glyceraldehyde-3-phosphate dehydrogenase; GMSCs: Gingival mesenchymal stem cells; LPL: Lipoprotein lipase; NANOG: Nanog homebox; OCN: Osteocalcin; OCT4: Octamer-binding transcription factor 4; OPN: Osteopontin; OSX: Osterix; PPAR-y: Peroxisome proliferater-activated receptor; SOX2: Sex-determining region Y-box 2

\section{Acknowledgements}

Not applicable.

\section{Authors' contributions}

$Y L$ and SM were responsible for the experimental design, manuscript revision, financial support, and manuscript approval. The collection, analysis, and interpretation of data, and manuscript writing were accomplished by $N H . L J, Y S, J D, L G$, and ZL were responsible for the collection, analysis, and interpretation of data. All authors approved the final manuscript.

\section{Funding}

This work was supported by grants from the National Nature Science Foundation of China (81470751 to YL, 81600891 to LG), the Beijing Municipal Administration of Hospitals Clinical Medicine Development of Special Funding Support (ZYLX201703 to YB), the Beijing Baiqianwan Talents Project (2017A17 to YL), the Beijing Municipal Administration of Hospitals' Ascent Plan (DFL20181501 to YL), the Beijing Municipal Science \& Technology Commission(Z16100000516203 to LG), the Beijing Municipal Administration of Hospitals' Youth Program (QML20181501), and the Beijing Dongcheng Excellent Talent (to $L G$ ).

\section{Availability of data and materials}

All data can be attained from corresponding authors.

\section{Ethics approval and consent to participate}

Animal experiments were approved by the Beijing Stomatological Hospital, Capital Medical University (Ethical Committee Agreement, Beijing Stomatological Hospital Ethics Review No. KQYY-201710-001).

\section{Consent for publication}

Not applicable.

\section{Competing interests}

The authors declare that they have no competing interests.

\section{Author details}

'Laboratory of Tissue Regeneration and Immunology and Department of Periodontics, Beijing Key Laboratory of Tooth Regeneration and Function Reconstruction, School of Stomatology, Capital Medical University, Tian Tan Xi Li No.4, Beijing 100050, People's Republic of China. ${ }^{2}$ Department of Orthodontics, School of Stomatology, Capital Medical University, Beijing, People's Republic of China. ${ }^{3}$ Beijing Tiantan Hospital, Capital Medical University, Beijing, People's Republic of China.

Received: 30 October 2019 Revised: 13 January 2020 Accepted: 22 January 2020 Published online: 14 February 2020

\section{References}

1. Takahashi N. Oral microbiome metabolism: from "who are they?" to "what are they doing?". J Dent Res. 2015;94:1628-37.

2. Baker JL, Bor B, Agnello M, Shi W, He X. Ecology of the oral microbiome: beyond bacteria. Trends Microbiol. 2017;25:362-74

3. Hoare A, Marsh PD, Diaz PI. Ecological therapeutic opportunities for oral diseases. Microbiol Spectr. 2017;4:5.

4. Yamashita Y, Takeshita T. The oral microbiome and human health. J Oral Sci. 2017:59:201-6

5. Samaranayake L, Matsubara VH. Normal oral flora and the oral ecosystem. Dent Clin N Am. 2017;61:199-215.
6. Rosier BT, Marsh PD, Mira A. Resilience of the oral microbiota in health: mechanisms that prevent dysbiosis. J Dent Res. 2018;97:371-80.

7. Meulen TA, Harmsen H, Bootsma H, Spijkervet F, Kroese F, Vissink A. The microbiome-systemic diseases connection. Oral Dis. 2016;22:719-34.

8. Struzycka I. The oral microbiome in dental caries. Pol J Microbiol. 2014;63: 127-35.

9. Costalonga $M$, Herzberg MC. The oral microbiome and the immunobiology of periodontal disease and caries. Immunol Lett. 2014;162:22-38.

10. Wang L, Ganly I. The oral microbiome and oral cancer. Clin Lab Med. 2014; 34:711-9.

11. Michaud DS, Izard J. Microbiota, oral microbiome, and pancreatic cancer. Cancer J. 2014;20:203-6.

12. Sarao LK, Arora M. Probiotics, prebiotics, and microencapsulation: a review. Crit Rev Food Sci Nutr. 2017;57:344-71.

13. Janczarek M, Bachanek T, Mazur E, Chałas R. The role of probiotics in prevention of oral diseases. Postepy Hig Med Dosw. 2016;70:850-7.

14. Matsubara VH, Bandara HM, Ishikawa KH, Mayer MP, Samaranayake LP. The role of probiotic bacteria in managing periodontal disease: a systematic review. Expert Rev Anti-Infect Ther. 2016:14:643-55.

15. Kasuya A, Tokura Y. Attempts to accelerate wound healing. J Dermatol Sci. 2014;76:169-72.

16. Lindley LE, Stojadinovic O, Pastar I, Tomic-Canic M. Biology and biomarkers for wound healing. Plast Reconstr Surg. 2016;138:18S-28S.

17. Burkhardt R, Lang NP. Influence of suturing on wound healing. Periodontol 2000. 2015:68:270-81.

18. Harrison JW. Healing of surgical wounds in oral mucoperiosteal tissues. J Endod. 1991;17:401-8.

19. Tsai HW, Wang PH, Tsui KH. Mesenchymal stem cell in wound healing and regeneration. J Chin Med Assoc. 2018;81:223-4.

20. Park SR, Kim JW, Jun HS, Roh JY, Lee HY, Hong IS. Stem cell secretome and its effect on cellular mechanisms relevant to wound healing. Mol Ther. 2018:26:606-17

21. Wu Y, Chen L, Scott PG, Tredget EE. Mesenchymal stem cells enhance wound healing through differentiation and angiogenesis. Stem Cells. 2007: 25:2648-59

22. Su Y, Chen C, Guo L, Du J, Li X, Liu Y. Ecological balance of oral microbiota is required to maintain oral mesenchymal stem cell homeostasis. Stem Cells. 2018:36:551-61.

23. Li X, Guo L, Liu Y, Su Y, Xie Y, Du J, Zhou J, Ding G, Wang H, Bai Y, Liu Y. MicroRNA-21 promotes osteogenesis of bone marrow mesenchymal stem cells via the Smad7-Smad1/5/8-Runx2 pathway. Biochem Biophys Res Commun. 2017:493:928-33.

24. Haneklaus M, Neill LA. NLRP3 at the interface of metabolism and inflammation. Immunol Rev. 2015;265:53-62.

25. Elliott E, Sutterwala FS. Initiation and perpetuation of NLRP3 inflammasome activation and assembly. Immunol Rev. 2015;265:35-52

26. Zhong Z, Sanchez E, Karin M. Autophagy, NLRP3 inflammasome and autoinflammatory/immune diseases. Clin Exp Rheumatol. 2016;34:12-6.

27. Jiang $\mathrm{H}, \mathrm{He} H$, Chen $Y$, Huang W, Cheng J, Ye J, Wang A, Tao J, Wang C, Liu $\mathrm{Q}$, et al. Identification of a selective and direct NLRP3 inhibitor to treat inflammatory disorders. J Exp Med. 2017:214:3219-38.

28. Teramachi J, Inagaki Y, Shinohara H, Okamura H, Yang D, Ochiai K Baba $R$, Morimoto $H$, Nagata T, Haneji T. PKR regulates LPS-induced osteoclast formation and bone destruction in vitro and in vivo. Oral Dis. 2017;23:181-8

29. Inagawa H, Kohchi C, Soma G. Oral administration of lipopolysaccharides for the prevention of various diseases: benefit and usefulness. Anticancer Res. 2011;31:2431-6.

30. Wade WG. The oral microbiome in health and disease. Pharmacol Res. 2013; 69:137-43.

31. Demmitt BA, Corley RP, Huibregtse BM, Keller MC, Hewitt JK, McQueen MB, Knight R, McDermott I, Krauter KS. Genetic influences on the human oral microbiome. BMC Genomics. 2017;18:659

32. Bozkurt SB, Hakki SS, Hakki EE, Durak Y, Kantarci A. Porphyromonas gingivalis lipopolysaccharide induces a pro-inflammatory human gingival fibroblast phenotype. Inflammation. 2017:40:144-53.

33. Tang J, Wu T, Xiong J, Su Y, Zhang C, Wang S, Tang Z, Liu Y. Porphyromonas gingivalis lipopolysaccharides regulate functions of bone marrow mesenchymal stem cells. Cell Prolif. 2015;48:239-48.

34. Basso FG, Soares DG, Pansani TN, Turrioni AP, Scheffel DL, Costa CA Hebling J. Effect of LPS treatment on the viability and chemokine 
synthesis by epithelial cells and gingival fibroblasts. Arch Oral Biol. 2015;60:1117-21.

35. Tang X, Metzger D, Leeman S, Amar S. LPS-induced TNF-alpha factor (LITAF)-deficient mice express reduced LPS-induced cytokine: evidence for LITAF-dependent LPS signaling pathways. Proc Natl Acad Sci U S A. 2006; 103:13777-82

36. Jerala R. Structural biology of the LPS recognition. Int J Med Microbiol. 2007; 297:353-63.

37. Talarico TL, Casas IA, Chung TC, Dobrogosz WJ. Production and isolation of reuterin, a growth inhibitor produced by Lactobacillus reuteri. Antimicrob Agents Chemother. 1988;32:1854-8.

38. Talarico TL, Dobrogosz WJ. Chemical characterization of an antimicrobial substance produced by Lactobacillus reuteri. Antimicrob Agents Chemother. 1989;33:674-9.

39. Peng Q, Scharer S, Puhan Z. Production and stability of 3hydroxypropionaldehyde in Lactobacillus reuteri. Appl Microbiol Biotechnol. 2002;60:73-80

40. Lian D, Dai L, Xie Z, Zhou X, Liu X, Zhang Y, Huang Y, Chen Y. Periodontal ligament fibroblasts migration injury via ROS/TXNIP/NIrp3 inflammasome pathway with Porphyromonas gingivalis lipopolysaccharide. Mol Immunol. 2018;103:209-19.

41. Martinon F, Pétrilli V, Mayor A, Tardivel A, Tschopp J. Gout-associated uric acid crystals activate the NALP3 inflammasome. Nature. 2006;440:237-41.

42. Guimaraes MR, Aquino SG, Coimbra LS, Spolidorio LC, Kirkwood KL, Rossa C. Curcumin modulates the immune response associated with LPS-induced periodontal disease in rats. Innate Immun. 2012;18:155-63.

43. Compan V, Baroja-Mazo A, López-Castejón G, Gomez Al, Martínez CM, Angosto D, Montero MT, Herranz AS, Bazán E, Reimers D, et al. Cell volume regulation modulates NLRP3 inflammasome activation. Immunity. 2012;37: 487-500.

44. Loomer PM, Sigusch B, Sukhu B, Ellen RP, Tenenbaum HC. Direct effects of metabolic products and sonicated extracts of Porphyromonas gingivalis 2561 on osteogenesis in vitro. Infect Immun. 1994;62(4):1289-97.

\section{Publisher's Note}

Springer Nature remains neutral with regard to jurisdictional claims in published maps and institutional affiliations.

Ready to submit your research? Choose BMC and benefit from:

- fast, convenient online submission

- thorough peer review by experienced researchers in your field

- rapid publication on acceptance

- support for research data, including large and complex data types

- gold Open Access which fosters wider collaboration and increased citations

- maximum visibility for your research: over $100 \mathrm{M}$ website views per year

At $\mathrm{BMC}$, research is always in progress.

Learn more biomedcentral.com/submissions 